\title{
Seguimento de portadores de coriorretinopatia serosa central por meio da tomografia de coerência óptica
}

\author{
Evaluation of central serous chorioretinopathy with optical coherence tomography
}

\author{
Otacílio de Oliveira Maia Júnior ${ }^{1}$ \\ Alexandre Kazuo Misawa² \\ Beatriz Sayuri Takahashi ${ }^{3}$ \\ Fabio Konno ${ }^{4}$ \\ Mariana Lie Yamaguishi ${ }^{5}$ \\ Walter Yukihiko Takahashi ${ }^{6}$
}

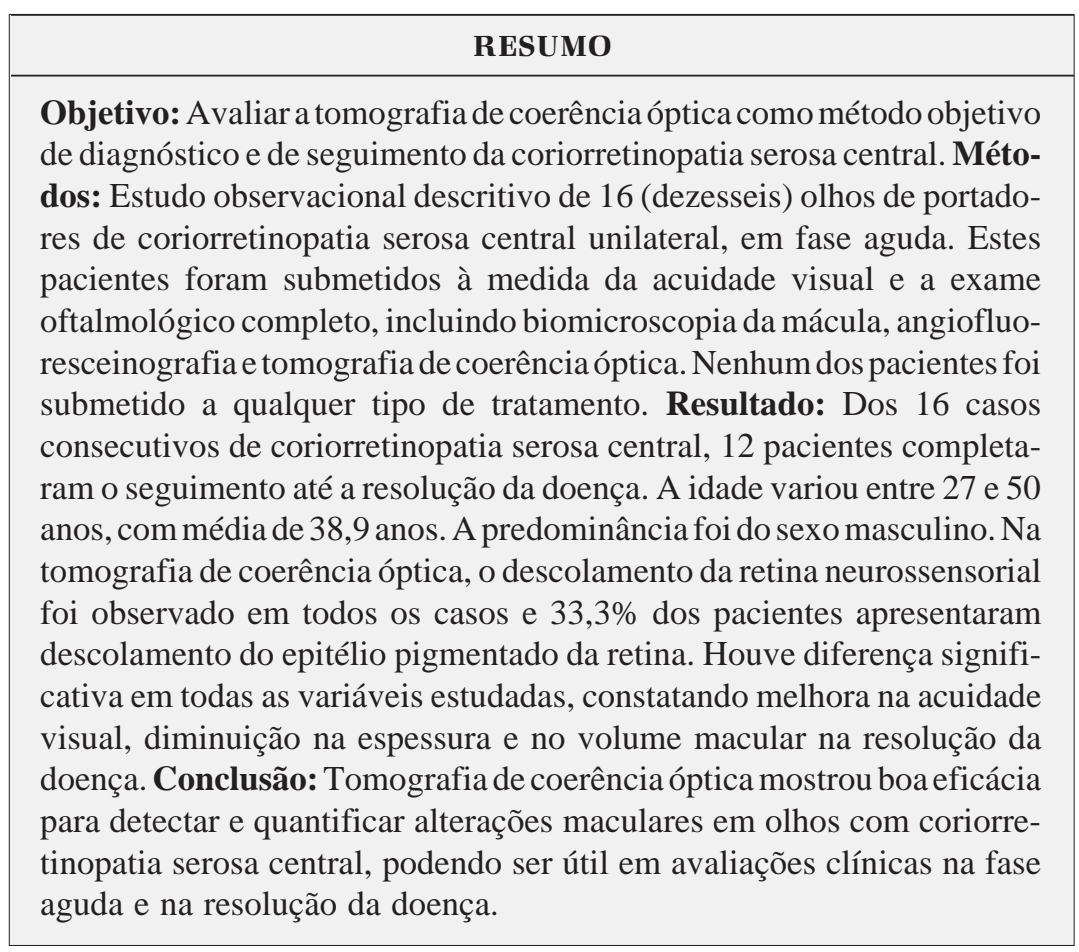

Descritores: Mácula lútea/anormalidades; Descolamento retiniano/diagnóstico; Descolamento retiniano/etiologia; Processamento de imagem assistida por computador; Angiofluoresceinografia; Tomografia/métodos

\section{INTRODUÇÃO}

A coriorretinopatia serosa central (CRSC) é caracterizada pelo descolamento seroso da retina na região macular, com resolução espontânea na maioria dos casos, sendo a fotocoagulação utilizada em situações selecionadas. A CRSC é mais comum na faixa etária jovem, dos 20-45 anos, principalmente no sexo masculino $(85 \%)$ e está associada com alguns fatores de risco como tensão emocional, gravidez e estresse cortisônico ${ }^{(1)}$. A angiofluoresceinografia (AFG) é o método utilizado para detectar o ponto de extravasamento do epitélio pigmentado da retina (EPR) e mostrar o acúmulo de contraste no espaço sub-retiniano(1).

A tomografia de coerência óptica, também conhecida pela sigla em inglês OCT, é um método diagnóstico que permite fornecer imagens de alta resolução das estruturas retinianas seccionadas transversalmente, sendo uma tecnologia não invasiva e sem necessidade de contato para a sua realização ${ }^{(2)}$. O Stratus $\mathrm{OCT}^{\mathrm{TM}}$, modelo mais avançado e disponível co- 


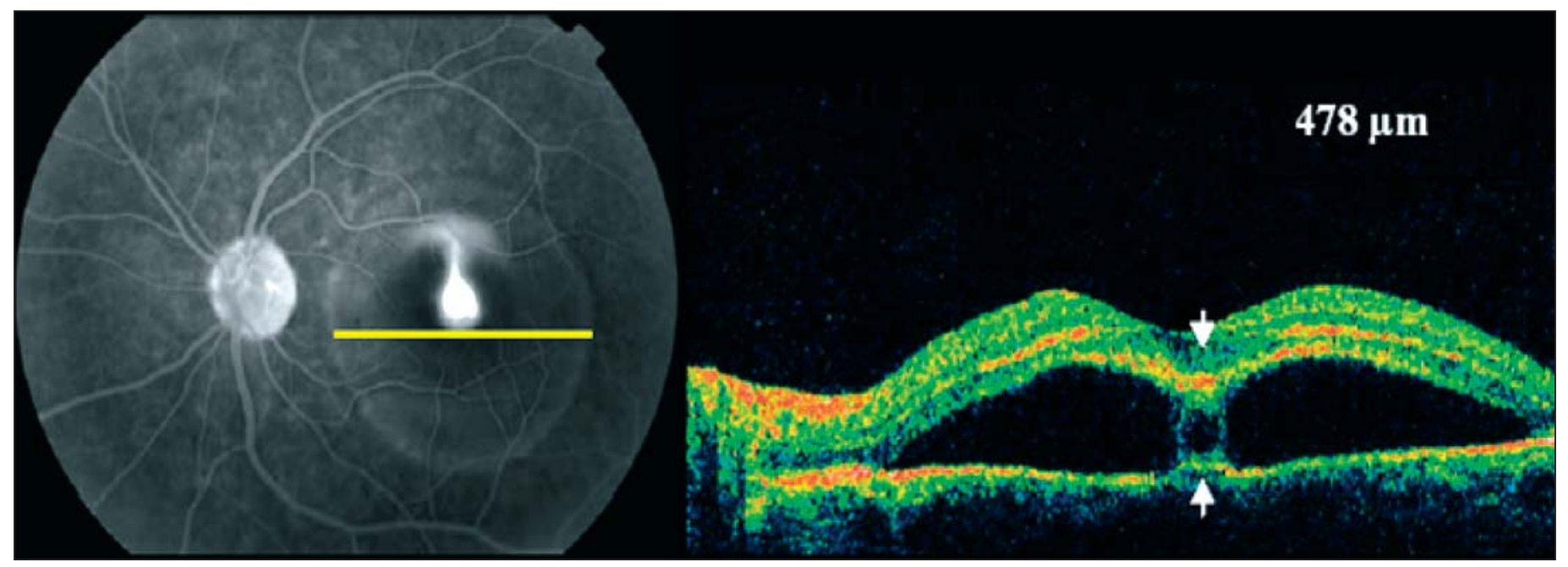

Figura 1 - Angiofluoresceinografia: observa-se ponto de vazamento ascendente em "fumaça de chaminé". Tomografia de coerência óptica: "scan" horizontal de $6,00 \mathrm{~mm}$ centrado na região foveal, evidenciando descolamento neurossensorial e ruptura do epitélio pigmentado da retina. Altura do descolamento no centro da região foveal foi de $478 \mu \mathrm{m}$.

mercialmente, possui uma resolução de aproximadamente 8 a $10 \mu \mathrm{m}^{(3-4)}$. Até pouco tempo atrás, a propedêutica para monitorar a resolução do descolamento neurossensorial da CRSC era limitada à biomicroscopia e à AFG. Com a introdução desta nova tecnologia, é possível diagnosticar casos de descolamentos mais planos, além de acompanhar a evolução e o tratamento $^{(5-6)}$.

A finalidade deste estudo é avaliar a tomografia de coerência óptica como método objetivo de diagnóstico e de monitoramento da resolução da CRSC.

\section{MÉTODOS}

Estudo prospectivo, observacional descritivo, de 16 (dezesseis) olhos de pacientes do Departamento de Oftalmologia do Hospital das Clínicas da USP, portadores de CRSC unilateral, em fase aguda.

O exame diagnóstico foi realizado por meio da biomicroscopia, AFG e pelo OCT "Stratus version 4.0 software" (Carl Zeiss Meditec).

O seguimento dos pacientes foi realizado até a reabsorção do fluido sub-retiniano no OCT. A queixa, o tempo de resolução e a acuidade visual foram determinados.

As imagens obtidas pelo OCT foram realizadas no programa de medida da espessura da retina (retinal thickness) e o cursor foi colocado manualmente no centro da fóvea quando a depressão foveal era visível ou na parte mais espessa no centro da mácula.

As variáveis quantitativas foram expressas em médias e desvios-padrão; as qualitativas calcularam-se frequiências absolutas e relativas. Para a comparação entre a fase aguda e a de resolução foi adotado o teste não-paramétrico de Wilcoxon ${ }^{(7)}$. O nível de significância utilizado para os testes foi de $5 \%$.

\section{RESULTADOS}

Dos 16 casos consecutivos de CRSC, 12 pacientes completaram o seguimento até a resolução da doença. O acometimento foi monocular em todos os pacientes, sendo o olho direito mais afetado $(66,7 \%)$ em relação ao esquerdo $(33,7 \%)$. A idade variou entre 27 a 50 anos, com média de 38,9. A predominância foi do sexo masculino com 9 pacientes $(75 \%)$ e $3(25 \%)$ eram do sexo feminino.

Quanto à sintomatologia, 91,2\% dos pacientes referiram diminuição da acuidade visual, 25\% metamorfopsia e 8,3\% escotoma relativo.

O diagnóstico foi firmado através da biomicroscopia e da AFG em todos os pacientes.

Quanto às alterações angiográficas, 66,7\% dos pacientes apresentaram ponto de vazamento padrão "mancha de tinta", $25 \%$ apresentaram hiperfluorescência por acúmulo de corante e 8,3\% apresentaram padrão em "fumaça de chaminé" (Figura 1).

No OCT o descolamento da retina neurossensorial foi observado em todos os casos (Figura 2), 33,3\% dos pacientes

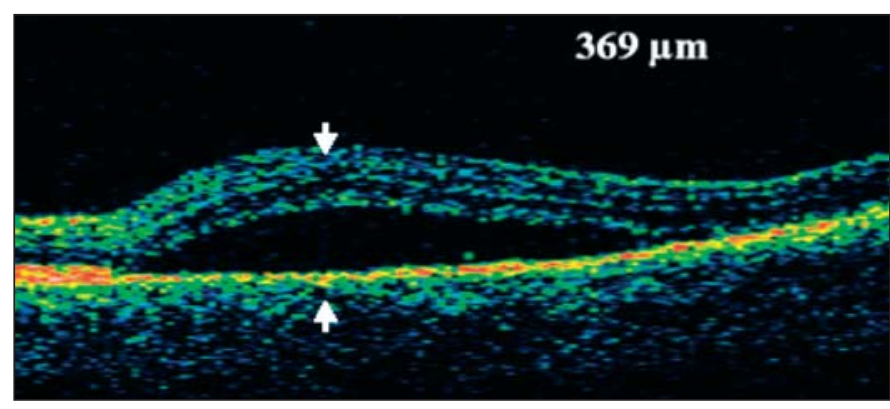

Figura 2 - Coriorretinopatia serosa central durante a fase aguda num paciente masculino de 42 anos. Imagem do Stratus $\mathrm{OCT}^{\mathrm{TM}}$ : descolamento seroso do neuroepitélio, com áreas de baixa refletividade intraretiniana. A espessura retiniana de $369 \mu \mathrm{m}$. Acuidade visual com correção, nesta fase, foi de $20 / 20$. 
apresentaram descolamento do EPR (Figura 3) e em 16,7\% foram evidenciadas estruturas de alta refletividade sub-retiniana (Figura 4). A espessura retiniana, na fase aguda da doença, variou de 320 a $721 \mu$ m (média de 493,7士122,9) e o volume macular variou de 7,63 a $20,84 \mathrm{~mm}^{3}$ (média de $11 \pm 4$ ).

A acuidade visual com melhor correção, na fase aguda, variou de 0,3 a 1,0 (média de 0,72 $\pm 0,25$ ).

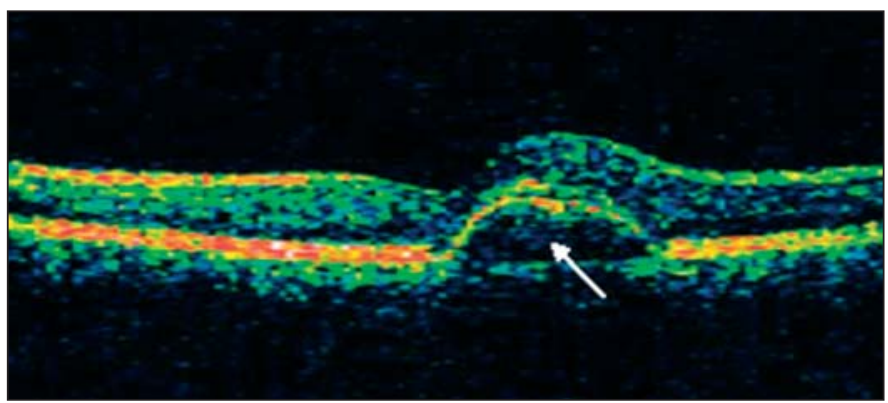

Figura 3 - Tomografia de coerência óptica: descolamento do epitélio pigmentado da retina (seta)
Nenhum dos pacientes teve indicação de qualquer tipo de tratamento. O tempo decorrido entre o diagnóstico da CRSC e a resolução do quadro variou de 30 a 120 dias (média de $85 \pm$ 26,63). Neste período, a espessura retiniana variou de 190 a

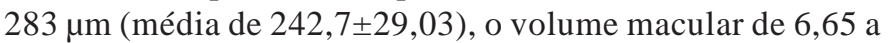
$8,38 \mathrm{~mm}^{3}$ (média de 7,63 $\pm 0,51$ ) (Figura 5) e a acuidade visual com melhor correção variou de 0,5 a 1,0 (média de $0,82 \pm 0,18$ ).

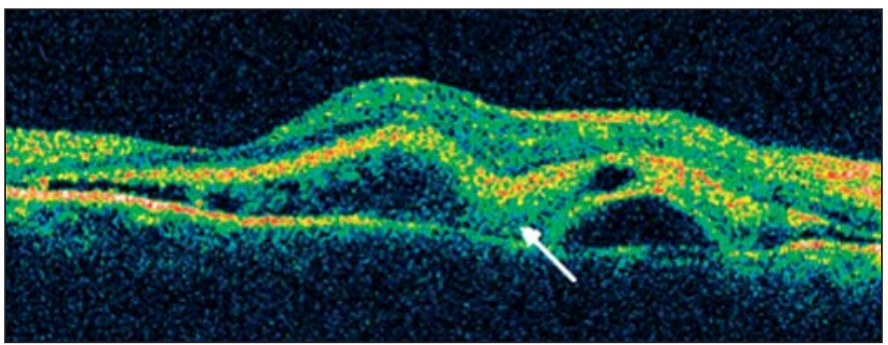

Figura 4 - Fase aguda da coriorretinopatia serosa central. Stratus $O C T^{\mathrm{TM}}$ demonstrando descolamento do epitélio pigmentado da retina e espaço sub-retiniano com estruturas de alta refletividade correspondentes a exsudatos fibrinosos (seta)

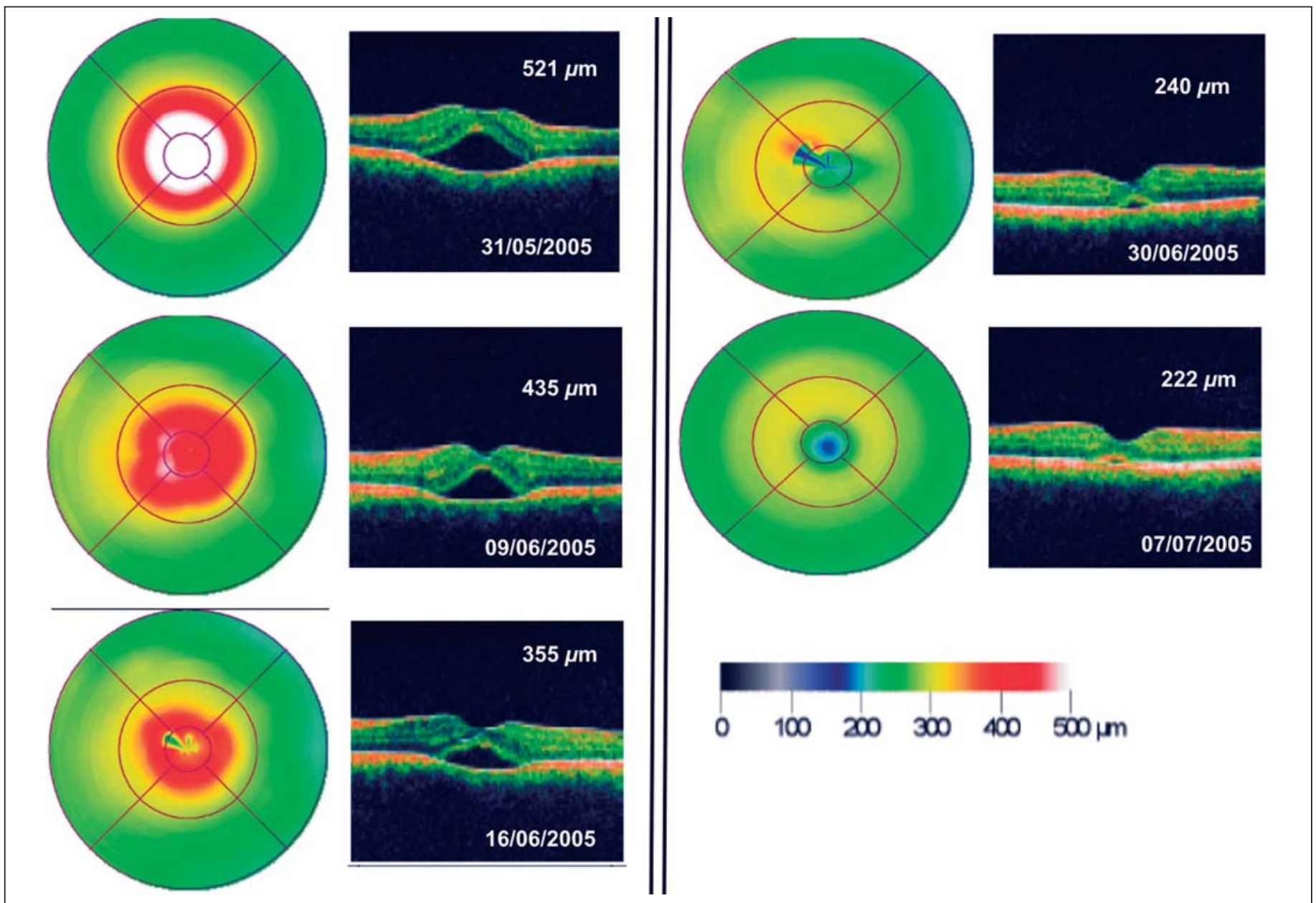

Figura 5 - Paciente feminina, 27 anos, com coriorretinopatia serosa central. Seqüências de "scans" do OCT evidenciando a significativa resolução do descolamento neurossensorial e uma persistência de fluido sub-retiniano residual na fóvea. Em todo seguimento, a paciente apresentou acuidade visual com correção de $20 / 20$, não sendo submetida a nenhum tratamento. 
Houve melhora estatisticamente significante na acuidade visual $(\mathrm{p}=0,031)$, redução da espessura $(\mathrm{p}<0,001)$ e no volume macular $(\mathrm{p}<0,001)$ na fase resolutiva da doença.

\section{DISCUSSÃO}

As novas tecnologias de imagem podem corroborar no diagnóstico e acompanhamento das doenças, trazendo um impacto nas pesquisas científicas e na prática clínica. O OCT possui atualmente diversas aplicabilidades clínicas. Na CRSC, os estudos iniciais começaram em 1995 com Puliafito e Hee, descrevendo uma série de casos avaliada pelo OCT, concluindo que este método é potencialmente útil como técnica diagnóstica não invasiva e capaz de avaliar quantitativamente o curso clínico do descolamento seroso da retina ${ }^{(5-6)}$. No presente estudo, as alterações da CRSC foram caracterizadas estruturalmente e quantificadas pela tomografia.

Descolamentos neurossensoriais pequenos na retina, difíceis de serem detectados pelo exame biomicroscópico e, até mesmo, pela AFG quando não há extravasamento do contraste, são passíveis de serem diagnosticados por meio do $\mathrm{OCT}^{(8)}$.

Recentemente, observou-se por meio do OCT que a espessura da retina neurossensorial descolada ficava significativamente mais espessa durante a fase aguda. Além disso, lesões branco-acizentadas sub-retinianas, observadas em alguns casos, seriam exsudatos fibrinosos que extravasariam da coróide, acumulando-se no espaço sub-retiniano e infiltrando a retina externa ${ }^{(9)}$. Há descrição de uma paciente gestante com CRSC na fase aguda apresentando exsudatos fibrinosos subretinianos que desapareceram após o parto ${ }^{(10)}$.

Em outro estudo, foi demonstrada uma relação entre os achados angiofluoresceinográficos com o OCT. As áreas de extravasamento evidenciadas pela AFG correspondem a áreas de alterações do EPR, seja como espessamentos ou descolamentos. As formas crônicas estão relacionadas com afilamento da retina neurossensorial e alturas maiores de descolamento do EPR. Nestes casos, a persistência do fluido sub-retiniano, presente na forma crônica ou recorrente, induziria alterações cistóides e afilamentos da retina ${ }^{(11)}$.

Nos casos de CRSC crônica, que persistem com baixa acuidade visual $(<20 / 200)$, podem apresentar degeneração macular cistóide, termo utilizado para descrever alterações císticas na retina interna, sem associação com extravasamento de contraste macular na AFG; pois quando isso ocorre, denomina-se de edema macular cistóide. Estas alterações, muitas vezes, não são diagnosticadas ao exame biomicroscópico e são detectáveis com a utilização do $\mathrm{OCT}^{(12)}$.

A relação da acuidade visual com as alterações observadas no OCT de alta resolução na CRSC foi recentemente discutida por Piccolino et al., observando que existia uma melhora da acuidade visual quando a camada de fotorreceptores estava preservada neste exame, o que não acontecia quando esta camada estava atrófica ${ }^{(13)}$. Neste estudo foram excluídos pacientes com atrofia do EPR subfoveal e degeneração cística da mácula, as quais poderiam também diminuir a acuidade visual $^{(12)}$. Outro trabalho que associa a acuidade visual com alterações anatômicas no OCT constatou que olhos com acuidade visual pior apresentavam espessura foveal mais fina que $o$ olho contralateral, também foi relacionado com a dificuldade de identificação da membrana limitante externa e a demarcação entre o corpo e o segmento externo dos fotorreceptores ${ }^{(14)}$.

\section{CONCLUSÃO}

O Stratus $\mathrm{OCT}^{\mathrm{TM}}$ demonstrou uma boa eficácia para detectar e quantificar alterações maculares em olhos com CRSC, podendo ser útil em avaliações clínicas na fase aguda da doença e na resolução. É um exame não invasivo, de fácil execução e interpretação, podendo ser incorporado ao arsenal propedêutico no seguimento destes doentes.

\section{ABSTRACT}

Purpose: To evaluate optical coherence tomography as an objective diagnostic and follow-up method for central serous chorioretinopathy. Methods: Observational descriptive study of 16 (sixteen) eyes of 16 patients with unilateral acute central serous chorioretinopathy. These patients had their visual acuity measured and complete ophthalmological examination was performed, including macular slit lamp examination, angiofluoresceinography and optical coherence tomography. No treatment was given to any patient. Results: Of the 16 central serous chorioretinopathy eyes, 12 patients completed follow-up until total resolution of their disease. Age varied between 27 and 50 years, with mean age of 38.9 years. Most patients were male. On optical coherence tomography, neurosensorial retinal detachment was observed in all cases and $33.3 \%$ had retinal pigmented epithelium detachment. There was a significant difference regarding all studied variables. Visual acuity was better, macular thickness and volume diminished when the disease resolved. Conclusion: Optical coherence tomography showed good efficacy to detect and measure macular changes in eyes with central serous chorioretinopathy, being useful in clinical evaluation during the disease's acute period and resolution.

Keywords: Macula lutea/abnormalities; Retinal detachment/ diagnosis; Retinal detachment/etiology; Image processing, computer-assisted; Fluorescein angiography; Tomography/methods

\section{REFERÊNCIAS}

1. Serracarbassa PD. Coroidoretinopatia central serosa. Arq Bras Oftalmol. 2002; 65(3):385-9.

2. Huang D, Swanson EA, Lin CP, Schuman JS, Stinson WG, Chang WM, et al. Optical coherence tomography. Science. 1991;254(5035):1178-81.

3. Jaffe GJ, Caprioli J. Optical coherence tomography to detect and manage retinal disease and glaucoma. Am J Ophthalmol. 2004;137(1):156-69. 
4. Hee MR, Izatt JA, Swanson EA, Huang D, Schuman JS, Lin CP, et al. Optical coherence tomography of the human retina. Arch Ophthalmol. 1995;113(3):325-32.

5. Puliafito CA, Hee MR, Lin CP, Reichel E, Schuman JS, Duker JS, et al. Imaging of macular diseases with optical coherence tomography. Ophthalmology. 1995;102(2):217-29.

6. Hee MR, Puliafito CA, Wong C, Reichel E, Duker JS, Schuman JS, et al. Optical coherence tomography of central serous chorioretinopathy. Am J Ophthalmol. 1995;120(1):65-74.

7. Rosner B. Fundamentals of biostatistics. 2nd ed. Boston: PWS; 1986.

8. Wang M, Sander B, Lund-Andersen H, Larsen M. Detection of shallow detachments in central serous chorioretinopathy. Acta Ophthalmol Scand. 1999;77(4): 402-5.

9. Iida T, Hagimura N, Sato T, Kishi S. Evaluation of central serous chorioretinopathy with optical coherence tomography. Am J Ophthalmol. 2000;129(1):16-20.
10. Rezai KA, Eliott D. Optical coherence tomographic findings in pregnancyassociated central serous chorioretinopathy. Graefes Arch Clin Exp Ophthalmol. 2004;242(12):1014-6.

11. Montero JA, Ruiz-Moreno JM. Optical coherence tomography. Characterization of idiopathic central serous chorioretinopathy. Br J Ophthalmol. 2005;89(5): 562-4.

12. Iida T, Yannuzzi LA, Spaide RF, Borodoker N, Carvalho CA, Negrão S. Cystoid macular degeneration in chronic central serous chorioretinopathy. Retina. 2003;23(1):1-7; quiz 137-8.

13. Piccolino FC, de la Longrais RR, Ravera G, Eandi CM, Ventre L, Abdollahi A, et al. The foveal photoreceptor layer and visual acuity loss in central serous chorioretinopathy. Am J Ophthalmol. 2005;139(1):87-99.

14. Eandi CM, Chung JE, Cardillo-Piccolino F, Spaide RF. Optical coherence tomography in unilateral resolved central serous chorioretinopathy. Retina. 2005;25(4):417-21.

\section{Congresso Internacional de Catarata e Cirurgia Refrativa}

V Congresso Internacional da Sociedade Brasileira de Administração em Oftalmologia

\section{1 de Maio a 3 de Junho de 2006 BELO HORIZONTE - MG}

\section{Informações sobre os Eventos}

IX Congresso Internacional de Catarata e Cirurgia Refrativa

Tel.: (31) 3226-3654 - Telefax: (31) 3274-6432

E-mail: congresso@catarata-refrativa.com.br

Home-page: www.catarata-refrativa.com.br

\section{Congresso Internacional da Sociedade Brasileira de} Administração em Oftalmologia

$$
\text { Tel.: (21) 2285-6052 }
$$

E-mail: sbao@sbao.com.br - Home-page: www.sbao.com.br 